\title{
Cuerpo incorpóreo: la representación de los fenómenos preternaturales como criterio de estudio
}

\author{
Non-Embodied Body: Representing Preternatural \\ Phenomena as Study Standard
}

ALMA LÓPEZ VALE*

\begin{abstract}
Resumen: Los fantasmas y fenómenos preternaturales han estado presentes en nuestra sociedad desde tiempos ancestrales. Su existencia se vuelve problemática en el tránsito a la Modernidad, época de la representación, alcanzando su cénit en el siglo XIX. En el presente trabajo exploramos cómo los fantasmas han sido descritos mediante un análisis y clasificación de sus vestimentas, planteando la necesidad misma de que se aparezcan con ropa y sus implicaciones. La representación, entonces, se vuelve criterio para el estudio ontológico, fenomenológico y sociológico de estos fenómenos.

Palabras clave: preternatural, fantasma, moda, representación, vestimenta.
\end{abstract}

\begin{abstract}
Ghosts and preternatural phenomena have been occurring in our society since immemorial times. However, during Modernity, the age of representation, their existence became a problematic issue, reaching its zenith during XIX century. In this paper we will explore how ghosts have been described through an analysis and classification of their clothes. We will also study the necessity of such a thing, i.e. why ghosts are seen with clothes and what this means. Representation, then, became criteria for an ontological, phenomenological as well as sociological study of preternatural phenomena.
\end{abstract}

Keywords: Preternatural, Ghost, Fashion, Representation, Clothes.

\section{A modo de presentación}

El estudio del cuerpo bajo el punto de vista de la filosofía puede abordarse desde múltiples ángulos, y en alusión a las sucesivas épocas históricas. Nos situaremos en un

Fecha de recepción: 09/06/2016. Fecha de aceptación: 06/09/2016.

* Investigadora contratada pre-doctoral FPI-UNED, Departamento de Filosofía de la UNED. Las principales líneas de investigación de la autora se refieren a los fenómenos extraños desde la Modernidad, atendiendo para ello a diferentes perspectivas, entre las que sobresalen los fantasmas y apariciones y la historia de las mujeres. Entre sus publicaciones más recientes se encuentran: López Vale, Alma (2016): «La constitución de la identidad a través de la relación con el fantasma», Cinta Moebio, $n^{\circ}$ 56, pp. 197-213; López Vale, Alma (2015): «Diario de una loca: la escritura como transgresión», en M. Martín Clavijo, Locas. Escritoras y personajes femeninos cuestionando las normas, Arcibel, Sevilla, pp. 245-263; y López Vale, Alma (2015): «Espacios espectrales: una relectura del mito del más allá como un conocimiento del más acá», en O. Cattedra, Mito e Historia III: el umbral del espacio, FASTA, Argentina, pp.187-202. Contacto: alma.1v.12@gmail.com 
ámbito un tanto diferente con la esperanza de, quizás, aportar un nuevo enfoque a esta multidimensionalidad corporal: los «cuerpos incorpóreos» como modo de expresión ideológica de cada época.

Delimitaremos temporalmente la investigación, que abarcará desde la Modernidad en adelante, fijándose muy especialmente en el siglo XIX por ser el momento de mayor apogeo de los fenómenos preternaturales. Por preternatural entendemos un fenómeno inusual que no parece natural, que no parece pertenecer o tener una explicación física. Nos referimos a un fenómeno que está fuera del curso ordinario de la naturaleza tal y como la conocemos y explicamos como humanos; un fenómeno que resulta extraño para nosotros en tanto seres limitados y para nuestras teorías científicas vigentes, que no disponen de las herramientas para una explicación satisfactoria (James, 1987, 316).

Con esta definición de «preternatural» nos acercamos a un conjunto de fenómenos y creencias místicas, religiosas, pero también populares y lo que hoy denominaríamos fenómenos paranormales. Con «preternaturales» denotamos que no nos referimos necesariamente a hechos del «más allá» (como la visión de fallecidos), sino también de nuestro «más acá», como el fenómeno de las duplicaciones o dobles, en el sentido relatado, por ejemplo, por Dostoievski en la obra homónima (1985).

La Modernidad supone una ruptura con la tradición de las creencias post-mortem, pasando de lo sobrenatural a lo preternatural. Es también momento del nacimiento del sujeto como individuo representacional (Foucault, 1984). En este sentido, tal y como Turró (1985) ha puesto de manifiesto, durante el Medievo...

...la existencia de leyes naturales rígidas supone que los prodigios o acontecimientos que se salen de la ley no pueden tener una causa natural, sino sobrenatural, lo que equivale a decir divina o diabólica. En cualquiera de ambos casos se está sosteniendo la existencia real de las potencias espirituales (Turró, 1985).

Esta creencia incuestionada se verá radicalmente transformada en la Modernidad, momento de surgimiento de la necesidad de preguntarse por la naturaleza de estos entes que, además, dejaban de estar asociados a instancias sobrenaturales como Dios, el demonio o los ángeles; y pasando a responder ante la imagen del mundo humana como representación (Heidegger, 1996).

Si durante el Medievo el estatuto ontológico de lo denotado por las palabras no suponía un problema puesto que cada significante tenía su referencia; en la Modernidad se abre una brecha entre ambas cuestiones, no vinculándose necesariamente el referente con lo referido o no existiendo lo referido de modo real. En la Época Medieval la palabra «unicornio» refería, apuntaba, a un ente, como también lo hacían los vocablos «demonio», «libro» ó «árbol». El paso a la Época Moderna constituye una ruptura en la que cada término puede o no contar con una referencia. En este marco, entonces, los fantasmas, antes incuestionados, se vuelven un problema de estudio: ¿qué entidad tienen? ¿De dónde surgen? ¿Por qué no los vemos todos?

\section{Un fenómeno complejo: el punto de vista subjetivo}

Las cuestiones anteriores nos remiten a lo que podríamos llamar el ámbito de la «imaginería mental», en tanto «es un término que hace referencia a las representaciones mentales 
que dan la experiencia de percepción sin la presencia de un estímulo sensorial aferente, por lo cual podemos considerarlo un fenómeno subjetivo» (Tamayo, 2014, 40). Aludimos, entonces, a la representación psicológica individual de estos fenómenos, la fenomenología de las visiones de fantasmas. Con ello se pretende poner de manifiesto cómo las características ideológicas, pero también sociológicas están nutriendo y alimentándose de estas representaciones.

La problemática a abordar, entonces, puede ser formulada como sigue: ¿a qué se deben los cambios en la representación de estos «seres» inmateriales? Sin entrar en su estatus ontológico -y de ahí la cautela al hablar de «seres»-, pues esta vía se nos antoja como muerta, sin solución: pretendemos estudiar su carácter más estético y psicológico. Para ello, debemos pensar, como se ha reflejado en el título de la ponencia, que estamos tratando un tipo de cuerpos incorpóreos. Las narraciones de este tipo de encuentros suelen ir acompañadas de una descripción en términos corpóreos de los «seres» que se aparecen o con los que se contacta cuando se trata, claro está, de apariciones visuales (las más comunes, por otra parte). Entre estas descripciones, ha de plantearse: ¿por qué algunos de esos «fantasmas» van vestidos de blanco, otros como un día normal y en otros casos solo podemos ver la luz que los rodea? ¿Se debe a que estamos ante la aparición «real» de personas fallecidas, por ejemplo? En este caso, ¿debería aparecerse con el atuendo del momento de la muerte o, por el contrario, con la vestimenta funeraria?

Si bien con la explosión cinematográfica todas las opciones representativas parecen estar cubiertas de algún modo (Clarke, 2010), la asociación entre fantasma y camisón o sábana blanca sigue estando presente. La vinculación entre espectros y la vestimenta blanca solo en la cultura occidental cuenta con más de una posibilidad, desde remontarse al sudario en el que Jesús fue envuelto, que era de ese mismo color; la palidez de los muertos que llevaría a la asociación de sus apariciones con el blanco; así como la creencia medieval de que aquellos que eran condenados a vagar por la tierra vestían con mantos blancos (Web: «Mujeres de blanco»).

El análisis de estas manifestaciones cinematográficas, aunque interesante, excede nuestros límites de tratamiento de la individualidad. Con ello no se pretende negar la dependencia bidireccional entre persona y época (Torregosa, 2010), pero dejaremos, por el momento, el cine puesto que construye él mismo las imágenes que nos son dadas como espectadores, siendo un medio de dación de imágenes externas y no correspondiéndose a la imaginería mental que será eje de nuestro estudio. Esta imaginería mental se plantea como base para tratar este aspecto raramente enfocado como criterio de decisión de la realidad de estos fenómenos. Hemos de poner sobre aviso al lector que por «realidad» entendemos implicaciones o consecuencias psicológicas y no presencia ontológica, pues esta decisión va más allá de los límites de nuestro entendimiento.

\section{Los fenómenos preternaturales}

Los fenómenos preternaturales han sido una constante a lo largo del tiempo y pueden ser considerados universales (Morin, 2003). En la tradición occidental este fenómeno fundamental se plantea con anterioridad a la Antigua Grecia (los primeros registros concretos datan de Mesopotamia, aunque se podría discutir si ya las pinturas rupestres y representaciones más primitivas son ejemplos de representaciones de esta naturaleza; véase Scotti, 2013). 
Los fantasmas forman parte de una creencia humana ancestral. A lo largo de las épocas han estado más en boga o menos, siendo especialmente importantes en la sociedad decimonónica. Cuando hablamos de fantasmas estamos ante un subconjunto de los fenómenos preternaturales, es decir, que están tanto en el más-allá como en el más-acá, evitando la denominación jerárquica y en buena parte mística de sobre-naturales. Se ha optado por esta manifestación puesto que contiene algunos elementos especialmente relevantes para nuestros propósitos. El más sobresaliente de estos rasgos lo constituye el hecho de que se trata de manifestaciones de «seres» o fenómenos incorpóreos, no extensos, de fallecidos. Además, en tanto universal (Morin, 2003), podemos pensar que este conjunto de fenómenos posee una relevancia sustancial para la humanidad como conjunto. Nuestro estudio de estos fenómenos parte de esta consideración de fenómeno fundamental en tanto espacio y temporalmente universal.

Estamos ante un fenómeno complejo, pues no existe consenso acerca de su denominación. A lo largo del tiempo y por diferentes motivos, como la propia evolución del lenguaje, las connotaciones religiosas de algunos términos y los usos de autores concretos han llevado a confundir más la terminología. En todo caso, sean denominados espíritus, espectros ó fantasmas -las tres más comunes- estamos refiriéndonos a apariciones de fallecidos que se manifiestan por medio de algún sentido -usualmente la vista, pero no sólo- y que no poseen cuerpo, no son extensos. Además, como grupo, no tienen un objetivo predeterminado, como pudiera ser la enseñanza moralizante o la metáfora política. De todos modos, suelen aparecerse por un motivo personal relevante o con alguna finalidad, pero no es este un rasgo único y común a todos los fantasmas, sino variable.

En esta característica, al igual que en la de la posesión del cuerpo, se contraponen los fantasmas a otros fenómenos preternaturales. Ejemplos de estos otras manifestaciones son el doble, ya mencionado; el zombi, una criatura bastante reciente y muy común actualmente; o el vampiro, cuya importancia se ha visto relevada en nuestros días. En todos estos casos estamos ante personajes no muertos: en el doble sería la duplicación del vivo, mientras que tanto zombi como vampiro se encuentran en una especie de limbo de no-vivos, pero nomuertos. Son, en todo caso, criaturas corpóreas y extensas.

Una vez definidos los fantasmas es necesario acotar el estudio temporalmente. Las particularidades históricas hacen que estos fenómenos se vuelvan filosóficamente relevantes en la Modernidad, contando con su máximo esplendor durante el siglo XIX. En épocas anteriores su estatuto ontológico no suponía un problema, sobre todo si pensamos en el entorno del cristianismo, en cuyo caso eran enviados sobrenaturales con existencia plena.

La Modernidad fue una época de profundos cambios sociales, económicos, religiosos y filosóficos que trajo consigo un nuevo sistema social, un nuevo régimen político y dio pie a un nuevo sistema económico; así como el paulatino camino hacia un pensamiento laico y una ciencia autónoma. Durante esta época se abre una brecha entre el sujeto y el mundo que dio lugar a la noción de representación (Foucault, 1984). Uno de los hitos fundamentales de estudio en el seno de esta ruptura serán, precisamente, los fenómenos extraños, como visiones de fantasmas que, paralelamente, cobran una relevancia y popularidad inusitadas. Ejemplo de ello lo constituye el místico y visionario sueco Emmanuel Swedenborg y su estudio por parte de Kant, reflejado en el ensayo Sueños de un visionario (Kant, 1987). 
Esta contraposición entre el Medievo y la ruptura ontológica de la Modernidad que hemos visto con Turró (1985), puede ser reforzada a través del siguiente fragmento en el que Heidegger relata el surgimiento de la representación: «la totalidad de lo existente se toma ahora de suerte que lo existente empieza a ser y solo es si es colocado por el hombre que representa y elabora», un hombre que es «medio de referencia de lo existente como tal» (Heidegger, 1996).

Estas características llevaron consigo a un auge paulatino de los fenómenos extraños o, más bien, de la necesidad de explicarlos. Las creencias que otrora fueran habituales como fe en lo sobrenatural se vuelven problemáticas al caer en el ámbito de lo preternatural, que debía de ser explicado. Este auge de los fenómenos extraños que transcurre en paralelo a la caída de Dios, tendrá su máximo apogeo en el siglo XIX, momento de la muerte de Dios (Lenz-Medoc, 1970).

En el seno de la sociedad decimonónica surge un grupo que se propondrá el estudio riguroso desde el punto de vista científico de los fenómenos extraños. Sus miembros fundarán en 1882 la Society for Psychical Research (SPR, como usualmente se la conoce). Entre ellos se encuentran personalidades relevantes de su época como dos Primer Ministro de Reino Unido (Lord Rayleigh y Arthur Balfour) ó, en su homóloga francesa, los premio Nobel Marie Curie, Charles Richet, Alfred Binet y Henri Bergson. En 1885 nacerá la sección americana $(A S P R)$, presidida por el también presidente de la American Psychological Association, William James.

El objetivo principal perseguido por los miembros de esta sociedad de investigación psíquica era el estudio riguroso desde un punto de vista científico de todo el conjunto de fenómenos extraños que no han tenido una explicación y que, por lo radical de extrañeza, han quedado fuera de los límites de la ciencia establecida, siendo marginados y tomándose, bien como creencias místicas, bien como meras supersticiones o, incluso, rasgos de una incipiente locura (James, 1987). Puede decirse, entonces, que:

...la $S P R$ se ha erigido como mecanismo para reunir la ciencia y el ocultismo en Inglaterra y América; y la creencia en que esta Sociedad cumple una función que aunque limitada está destinada a tener su importancia en la organización del conocimiento humano (James, 1987, 302).

Se establecieron diferentes comités encargados de aspectos concretos de lo psíquico, tales como la comunicación con otras mentes o «telepatía» (Myers, 1882); la visión o percepción de fenómenos extraños relacionados con lo espectral (Sidgwick et al., 1894); descubriendo un fenómeno como el de la aparición de personas a familiares o amigos íntimos en momentos críticos, como el momento de la muerte (Gurney et al., 1886). Esta última investigación fue desarrollada por Gurney, Myers y Podmore y recogida en la obra Phantasms of the Living (1886). En él desvelaron que se trataba de un fenómeno relativamente frecuente, habiendo sido tratado largamente como sobrenatural en el sentido de comunicaciones con el más allá, pero que se correspondía con el mundo de los vivos, el mundo real, y, entonces, con lo preternatural.

Se planteó en la Sociedad el motivo de tantos detalles del atuendo narrados por los visionarios. El problema a confrontar era por qué se veía modificada la representación de un 
aspecto tan banal en la aparición. ¿Se debía a que eran manifestaciones reales de espíritus? La moda, entonces, se planteó como posible criterio de decisión en una cuestión que llevaba una trayectoria de milenios. Si bien la importancia del atuendo, derivada de la cuestión de la representación, no tomaría relevancia hasta la Modernidad. No deja de resultar extraño que se hiciese hincapié en la descripción de estos detalles.

La cuestión que nos planteamos, entonces, es: ¿cómo se representan? ¿Cuál es su estética? ¿Existe una única representación que se mantiene en el tiempo o se transforma con el paso de los años? De ser así... ¿a qué se deben dichos cambios? Estas cuestiones resultaron relevantes para los miembros de la $S P R$ en tanto características de fenómenos muy en boga en su momento histórico.

No deja de resultar curioso que pueda existir algo así como una «moda para fantasmas», puesto que estamos hablando de seres incorpóreos, no extensos. Pensemos que en aquellos casos en los que se trata de manifestaciones de fallecidos, causa una mayor extrañeza el hecho de que se aparezcan con ropaje. Para los denominados «fantasmas de los vivos» (Gurney et al., 1886) la similitud de la descripción con la vestimenta que de facto portaba la persona aparecida en aquel momento lleva a establecer una duda seria de cara a su posible verdad. ¿Cómo, sino, es posible que se describa tan exactamente el atuendo del aparecido?

La indumentaria de las apariciones es uno de los hitos descriptivos más sobresalientes en las narraciones de visiones de fantasmas. La estética, entonces, se vuelve quizás representativa de las propias ideas de aquellos que están padeciendo la visión o quizás, la prueba definitiva de su existencia. La cuestión principal que se establece como criterio de decisión acerca de la veracidad de los relatos de apariciones, es: ¿para qué cambiar de atuendo? Y, lo que es más, ¿para qué tener uno?

\section{La representación de las visiones: diferentes estilos}

Partiendo del riguroso estudio emprendido por SPR en torno a los fenómenos espectrales, nos centraremos en lo que la gran escéptica Nora Sidwick denominó «ghosts of clothes» (Blum, 2006), pues le sorprendía que «viniesen» del más allá vestidos y fuese éste uno de los rasgos que más sobresalía a la hora de describir una visión. El hecho de que la secretaria de la sociedad fuese escéptica resulta de interés en tanto que su intención era más la de desenmascarar el fraude que la posibilidad de llegar a probar la existencia de fantasmas. Sus estudios, investigaciones, recensiones de otros trabajos y talante en general imprimió vigor en la sociedad y un carácter falsacionista, de alta calidad y rigurosidad científica (Emmons y Emmons, 2012).

Nuestra propuesta parte de la base de que el estudio de los fantasmas es relevante desde el punto de vista de los visionarios, independientemente de la existencia «real» o no de los espectros. Dicha importancia se justifica, tal y como el «Census of Hallucinations» (Sidgwick et al., 1894) mostró tras obtener más de 17000 respuestas en Reino Unido, por el gran número de personas de nuestra cultura y la gran diversidad de culturas que experimentan estos episodios. Es un intento de explicación de las visiones de fantasmas desde la búsqueda de los motivos que hacen que se aparezcan frente al análisis ingenuo y poco informativo usual, basado en buscar una explicación a la aparición desde el punto de vista del fantasma -conciencia intranquila, venganza... (Clarke, 2012). 
Dejando a un lado el reduccionismo que ha imperado (Sommer, 2014), realizaremos el análisis de la vestimenta de las apariciones. En relación a la moda, las visiones podrían ser clasificadas en tres grandes grupos: un primer conjunto de fantasmas «en la noche», que se corresponde con apariciones nocturnas con atuendo de dormir. Estas apariciones son usualmente nocturnas y de personas en el momento de su muerte, por lo que son llamadas «apariciones críticas» (Gurney et al., 1886). Un segundo tipo de fantasmas que visten «de luto» (Soctti, 2012), es decir, de negro, color asociado a la muerte y que representa generalmente una muerte prematura e injusta, dolorosa; estando el tercer grupo formado por aquellos cuyo atuendo es el habitual (Blum, 2006) o lo que coloquialmente denominamos «de diario». Estos fantasmas parecen no haberse percatado de su muerte y perseveran en sus acciones cotidianas, aquellas que más los representan. Existe, aún, un cuarto tipo de apariciones que podríamos catalogar como «trasparentes» que, pese a su denominación basada en los relatos de aquellos que las ven son nítidamente percibidas, a efectos prácticos para nuestro ensayo, podrían clasificarse bajo cualquiera de los tres grupos anteriores.

Pongamos algunos ejemplos de esta indumentaria fantasma, todos ellos de carácter «real», es decir, narrados como verídicos por las personas que los padecieron y recogidos como objeto de estudio por los cronistas. Es decir, no forman parte de obras literarias, novelas, películas u otras ficciones, sino que son experimentadas por personas como hechos, por lo que serían fenomenológicamente reales.

En primer lugar, la historia relatada una mujer que, de repente, se despertó durante la noche. Sintiéndose incómoda se sentó en la cama y vio a su hijo Joseph en la puerta con la cara cubierta de sangre. Vestía un sucio pijama. Ya por la mañana la mujer reunió a su familia para avisarlos de que vendrían malas noticias sobre Joseph, pero todos se rieron de ella diciéndole que no se preocupase, que había sido solo un sueño. Unos días más tarde, sin embargo, recibieron noticias de su hijo: había muerto en la colisión de dos vapores en el Mississippi, la noche y a la hora que se le había aparecido. Un hermano que estaba con él durante el trágico suceso lo relató de modo muy similar a la experiencia de la madre: la cara de Joseph había quedado cubierta de sangre tras la explosión, manchando el pijama que llevaba puesto en el momento de su muerte durante el cual llamaba a su madre (Blum, 2006).

Presentamos también el caso de la familia Despard, por el gran detalle de la descripción de la vestimenta de la mujer fantasma.

Todos en la familia comenzaron a sufrir apariciones, sobre todo Rosina, la hija mayor. Una noche oyó un ruido fuera de su habitación, pero cuando abrió la puerta no había nadie. Siguió la pista por el pasillo y miró hacia las escaleras, en lo alto, aunque todo estaba oscuro, vio a una mujer alta, vestida completamente de negro. Rosina observó a la figura bajar las escaleras y la siguió mientras su minúscula vela se lo permitió. Su descripción posterior de los hechos estaba repleta de detalles. La cara del fantasma estaba cubierta por un pañuelo y su mano izquierda se escondía en la manga de su vestido. La aparición no era transparente. Incluso parecía ser bastante sólida... (Canwell, Sutherland, 2007).

Algunos de estos relatos se producen en torno a lugares famosos, como el castillo de Windsor, rodeado de visiones de fantasmas, como el de Enrique VII o Ana Bolena. Ésta se aparece, no de modo terrorífico como sería portando su cabeza bajo el brazo, sino que los 
que la han visto afirman que está mirando por la ventana en el Dean's Cloister, siempre con un vestido largo, de color negro y un chal de encaje de la misma tonalidad sobre sus hombros (Canwell, Sutherland, 2007).

También están los ejemplos de fantasmas que se aparecen con su vestimenta habitual de vivos, como los vistos en castillos, lugares en los que son habituales las apariciones de fantasmas:

En Arundel Castle (Reino Unido) se manifiesta un joven que vaga por las habitaciones de los sirvientes que viste una túnica sin mangas. Otro ejemplo es el de Carisbrooke Castle, en dónde la alcazaba es visitada por una Dama de Gris, que viste una larga capa y va acompañada de cuatro perros. En el mismo castillo se relata la aparición de un joven con un chaleco marrón que a veces de deja ver en las proximidades del foso. Otra visión frecuente es la de una mujer con un vestido victoriano que es perseguida por las sombras de dos canes. También una aparición con un largo abrigo se aprecia a plena luz del día (Canwell, Sutherland, 2007).

Por último, me gustaría relatar un caso de una joven que tras haber sido dada por muerta -sin estarlo- es velada por su padre. En el trascurso del velatorio la chica se le aparece a su padre con su atuendo mortuorio: la mortaja. Tras experimentar la aparición el padre, asustado, recurre al médico, quién certifica la muy reciente muerte de la chica, que había estado en coma (Canwell, Sutherland, 2007).

Durante el siglo XIX, momento de máximo esplendor de las visiones y apariciones de fantasmas, se dieron tres posibles interpretaciones a esta cuestión: una partió de la negación absoluta tanto de los fantasmas, como del interés en el estudio de los mismos. Fue la posición sostenida por Stanley Hall (Kurtz, 1985), por ejemplo. En segundo lugar, estaría la posición de la propia Nora, que vería la vestimenta como prueba de la no existencia real de los fantasmas por su carácter superfluo para un espíritu venido de la muerte. Mostraría que se trata de una construcción del visionario y no un fenómeno real. Por último, algunos autores de la $S P R$, tras diversas pruebas, entrevistas y experimentos, afirmarían que este rasgo puede ser postulado como prueba de verificación y, en última instancia, de existencia real de los fantasmas (Gurney et al., 1986; Blum, 2006).

\section{A modo de conclusión: claves interpretativas}

Sin pretender entrar en cuestiones ontológicas que son callejones sin salida, es necesario señalar que estos estudios respondían a la necesidad de pruebas de la existencia de fantasmas tanto como a un intento de análisis del fenómeno desde la perspectiva psicológica del visionario, es decir, de los motivos de su visión y el intento de dilucidación de la compleja situación a partir de los detalles que relataba. Además, no debemos olvidarlo, los fantasmas eran en sí mismos una «moda» por aquel entonces, puesto que el intento de estudio de este fenómeno no se aleja de su momento social.

La cuestión última que se plantea es si, tal y como los miembros de la $S P R$-entre otros- hicieron, la moda de los fantasmas puede ser establecida como criterio. La respuesta es múltiple: por un lado será «no» si lo pensamos como criterio ontológico. Esta cuestión, en todo caso, no es central para nuestro estudio. La existencia o no existencia de fantasmas no cambia su función o importancia para aquellas personas que los ven. La relevancia del 
estudio, no obstante, permanece intacta, desde el momento en que estamos ante un problema antropológico fundamental y, todo apunta a que, universal. No debemos olvidar el horizonte tenso que se establece entre la vida y la muerte, quedando el fantasma en el punto medio, como transición entre ambos polos que parece útil para algunos, necesaria para otros y pertinente, en todo caso, para la humanidad como conjunto.

Por otro lado, la respuesta a si la «moda fantasma» puede ser considerada criterio bien pudiera ser afirmativa si lo que nos preocupa es buscar su significación tanto para aquellos que experimentan las visiones a título personal, individual, como su sentido social, como espejo de cada momento. Si es esta nuestra preocupación -como, en efecto, lo es- diremos que los fantasmas pueden considerarse un criterio de investigación de los rasgos psicológicos individuales y las características constitutivas de la sociedad temporalmente fijadas.

¿A qué nos referimos con rasgos psicológicos individuales? Nuestra pretensión no es -espero- en exceso pretenciosa, tal y como esta afirmación pudiese dar a entender. No estamos hablando de desarrollar una novedosa teoría psicológica de rasgos o categorías bajo las que albergar o clasificar a las personas. Los intentos que se han hecho en este sentido son, por lo que sabemos, más bien delicados (Madsen, 2014) tanto desde el punto de vista de la «ciencia» a la que defendían como desde el humano, llevando a estigmatizar, sino martirizar a ciertos sectores de la población (Goffman, 1998).

Nuestra pretensión va más allá de este reduccionismo, siendo al mismo tiempo más modesta. Se trata de buscar qué necesidades se esconden bajo los motivos que llevan a una persona a ser visionaria para intentar paliarlas. Entre ellas se encuentra el duelo o sufrimiento insoportable por la muerte de un ser querido; la necesidad de expresión de una subjetividad reprimida, como en el caso de muchas histéricas decimonónicas (Montiel, 2006); ó, por qué negarlo, la alucinación que puede ser o no síntoma de una enfermedad mental que haya de ser tratada, siendo también posible que se trate de un fenómeno no patológico (Johns et al., 1998).

En cuanto a los rasgos sociales, mantenemos que los fantasmas pueden ser expresión de las ideologías de cada momento (Ferrero, Roas, 2011), como así lo fueron los vampiros -metáfora de la aristocracia tendente a desaparecer- o los zombis -representación de la masa que se rebela (Martínez Lucena, Barraycoa, 2012).

Aunque como conjunto no posean una característica o finalidad concreta, las modificaciones y evoluciones de las apariciones de fantasmas nos dan pistas de la ideología que se esconde tras ellos. Si durante el siglo XVIII se representaban mayormente todavía como ángeles o experiencias místicas (Scotti, 2015), en el XIX fueron progresivamente «normalizándose», formando parte de la cotidianeidad. Estos rasgos propios de las creencias y tendencias de cada momento se observan en la indumentaria, por lo que podemos afirmar que es un criterio de examen social.

Concluyendo... ¿de qué hablamos cuando decimos moda de fantasmas? ¿Es de un mero atuendo exterior, unos meros ropajes que nos guardan del frío, nos alivian el pudor creado hacia los cuerpos desnudos o es una formulación de la identidad en su sentido fuerte? Pensemos que, a través de la relación de sujeto y fantasma, éste se conforma, se conoce y re-conoce debido, precisamente, a su vestido. Estamos, entones, no solo ante una percepción o experiencia «distorsionada» de la realidad, sino ante la constitución de las identidades a través de la moda. En un mundo tal, un mundo teatral, de la imagen, de la mera apariencia... 
¿qué problema supone que algunas personas crean en fantasmas o experimenten visiones de fallecidos? ¿No somos todos, acaso, meras imágenes especulares -espectrales- de lo que la propia sociedad está proyectando en nosotros?

\section{Bibliografía}

Canwell, Diane, Sutherland, Jonathan (2007): Ghosts of the World, Chartwell Books, Londres. Clarke, Roger (2012): A Natural History of Ghosts: 500 Years of Hunting for Proof, Penguin Books, Londres.

Clarke, Roger (2010): Story of the Scene: The inside scoop on famous moments in film, Londres, Methuen Drama/Bloomsbury Publishing.

Dostoievski, Feodor (1985): El Doble, Alianza, Madrid.

Emmons, Charles, Emmons, Penelope (2012): Science and Spirit: Exploring the Limits of Consciousness, Bloomington, IUniverse.

Ferrero, Ángel, ROAS, Saúl (2011): «El 'zombi' como metáfora (contra)cultural», Nómadas, n³2(4), pp. 97-220.

Foucault, Michel (1984): Las palabras y las cosas, Siglo XXI, Madrid.

Goffman, Erving (1998): Estigma: la identidad deteriorada, Amorrortu, Buenos Aires.

Gurney, Edmund, Myers, Frederick W.H., Podmore, Frank (1886): Phantasms of the Living, 2 vols, London, Trübner.

Heidegger, Martin (1996): «La época de la imagen del mundo», en Caminos de Bosque, Alianza, Madrid.

James, William (1987): «What Psychical Research Has Accomplished?» En Burkhardt, F. H., Bowers, F., Skrupskelis, I. K. (eds.), Works of William James, vol. 17, pp. 299-328.

Johns, Louise C., et al. (1998): «Ocurrence of Hallucinations in a Community Sample», Schizophrenia Research, n²9 (23), pp. 23-25.

Kant, Inmanuel (1987): Sueños de un visionario, UCA, Cádiz.

Kurtz, Paul (1985): A Skeptic's Handbook of Parapsychology, Prometheus Books, Nueva York.

Lenz-Medoc, Pedro (ed). (1970): La muerte de dios, Monte Avila, Caracas.

Madsen, Ole (2014): The Therapeutic Turn: How Psychology Altered Western culture, Routledge, Londres.

Martínez Lucena, Jorge, Barraycoa, Javier (2012): «El zombi y el totalitarismo: de Hannah Arendt a la teoría de los imaginarios», Imagonautas, $\mathrm{n}^{\circ} .2(2)$, pp. 97-118.

Montiel, Luis (2006): «Síntomas de una época: magnetismo, histeria y espiritismo en la Alemania romántica», Asclepio, $n^{\circ}$.LVIII(2), pp. 11-38.

Scotti, Massimo (2013): Storia degli spettri. Fantasmi, medium e case infestate fra scienza e letteratura, Feltrinelli, Milán.

Sidgwick Henry, et al. (1894): «Report on the Census of Hallucinations», Proceedings of the Society of Psychical Research, $\mathrm{n}^{\circ} 10$, pp. 25-422.

Sommer, Andreas (2014): «Psychical Research in the History and Philosophy of Science: An Introduction and Review», Studies in History and Philosophy of Biological and Biomedical Sciences, n48, pp. 38-45. 
Tamayo, Nathalie (2014): «Imaginería mental: neurofisiología e implicaciones en psiquiatría», Revista Colombiana de Psiquiatría, n43(1), pp. 40-46.

Torregrosa, Marta T. (coord.) (2010): Imaginar la realidad. Ensayos sobre la representación de la realidad en el cine, la televisión y los nuevos medios, Comunicación Social, Sevilla. Turró, Salvio (1985): Descartes: del hermetismo a la nueva ciencia, Anthropos, Barcelona. Web: «Mujeres de blanco» en Mitos, Monstruos y Leyendas», http://mitosmonstruosyleyendas.blogspot.com.es/2015/06/las-mujeres-de-blanco.html;consultado 22/05/2016. 
